\title{
A Study on Modelling the Superplastic Behaviour of Ti54M Alloy
}

\author{
Hosam Elrakayby ${ }^{1, a}$, Diego Gonzalez ${ }^{1, b}$, Ares Gomez-Gallegos ${ }^{1, ~ c}$, Paranjayee \\ Mandal ${ }^{1, d}$ and Nicola Zuelli ${ }^{1, e}$
}

${ }^{1}$ Advanced Forming Research Centre, University of Strathclyde, 85 Inchinnan Drive, Inchinnan, Renfrewshire PA4 9LJ, Scotland.

ahosam.elrakayby@strath.ac.uk, bdiego.gonzalez@strath.ac.uk, cares.gomez-gallegos@strath.ac.uk, dparanjayee.mandal@strath.ac.uk, en.zuelli@strath.ac.uk

Keywords: Superplastic Forming; Titanium; Ti54M; Finite Element Modelling

\begin{abstract}
Superplastic forming is a cost-effective process for manufacturing complex-shaped titanium parts. TIMETAL ${ }^{\circledR} 54 \mathrm{M}$ (Ti54M) is a titanium alloy that has been commercially available since 2003, however studies on modelling its superplastic behaviour are scarce in the literature. Finite element modelling can be used to enable the manufacturing of complex-shaped parts economically as the number of experimental trials can be reduced. This paper illustrates the implementation of a microstructuralbased model to predict the superplastic behaviour of Ti54M alloy during forming at elevated temperature. The parameters of the material model are derived in this work for the Ti54M alloy. A Matlab script has been developed for the calculation and calibration of the material model parameters based on material experimental data. The material model was implemented into the finite element commercial software Abaqus by means of a user-defined subroutine. The finite element calculations take into account also grain size evolution. Finally, a pressure profile was numerically calculated for forming a non-commercial part via superplastic forming targeting optimal conditions for the material.
\end{abstract}

\section{Introduction}

For more than fifty years, superplastic forming (SPF) has been used to manufacture complex-shaped parts mainly for aerospace and automotive industries [1]. Finite element modelling has a significant role in manufacturing these parts economically by saving the costs of experimental trials. Titanium $(\alpha+\beta)$ alloy Ti-6Al-4V (Ti64) is extensively used in SPF to form parts for aerospace applications due to its high strength to weight ratio and excellent corrosion resistance [2,3]. TIMETAL ${ }^{\circledR} 54 \mathrm{M}$ (Ti54M) is another titanium $(\alpha+\beta)$ alloy that has almost the same mechanical properties of Ti64 alloy but superior machinability [4]. The $\beta$-transus temperatures of Ti54 and Ti6-4 are $966^{\circ} \mathrm{C}$ and $995{ }^{\circ} \mathrm{C}$, respectively [5]. Therefore, it is expected that Ti54M alloy can be formed via superplastic forming at temperatures lower than that required to form Ti64 alloy [6].

Although Ti54M alloy has the potential to replace Ti64 alloy in superplastic forming applications, the literature lacks studies on modelling the superplastic behaviour of Ti54M alloy. The present work utilizes a microstructural-based power-law model to predict the superplastic behaviour of Ti54M alloy at $815{ }^{\circ} \mathrm{C}$ and compare it to the superplastic behaviour of Ti64 alloy at $925^{\circ} \mathrm{C}$. These temperatures were reported to be optimal for Ti54M and Ti64 alloys based on strain rate sensitivity values and uniform deformation conditions $[7,8]$.

The parameters of the material model have been calculated based on material experimental data by means of a developed Matlab script for Ti54M and Ti64 alloys. The material experimental data used in this work are reported in the literature [7,8]. Mandal et al. [7] studied the grain size evolution of Ti54M and Ti64 alloys under superplastic deformation for various test conditions. Gomez-Gallegos et al. [8] 
investigated the mechanical and superplastic properties of Ti54M and Ti64 alloys at various temperatures via high temperature tensile tests.

Finally, finite element calculations for grain size evolution are reported in this study. These data were obtained from the calibrated material model simulating the SPF process of a non-commercial part for Ti54M alloy at $815^{\circ} \mathrm{C}$ and Ti64 alloy at $925^{\circ} \mathrm{C}$. Pressure profiles and the ratio between the maximum strain rate in the material to the target strain rate of $5 \times 10^{-4} \mathrm{~s}^{-1}$ (this target strain rate is imposed to control the forming process) are also numerically calculated.

\section{Constitutive Equations}

For numerical modeling of the SPF process, a microstructural-based model was used to represent the material behaviour. This included two aspects as follows:

Grain Growth. The constitutive Eq. 1 was employed to model the grain size evolution during SPF process $[9,10]$.

$$
\dot{d}=\dot{d}_{\text {static }}+\dot{d}_{\text {dynamic }}
$$

The static grain growth $\dot{d}_{\text {static }}$ in Eq. 2 captures the grain growth by atomic diffusion due to the high temperatures in the absence of deformation conditions. The dynamic grain growth $d_{d y n a m i c}$ in Eq. 3 is related to the deformation of the material.

$$
\begin{aligned}
& \dot{d}_{\text {static }}=\frac{\rho}{d^{\mu_{1}}} \\
& \dot{d}_{\text {dynamic }}=\frac{\varphi}{d^{\mu_{2}}} \dot{\varepsilon}
\end{aligned}
$$

Where $\rho, \mu_{1}, \varphi$ and $\mu_{2}$ are temperature-dependent material parameters. $\dot{\varepsilon}$ is the strain rate.

Mechanical Behaviour. The visco-plastic response of the material is expressed by a power-law based model in Eq. 4.

$$
\dot{\varepsilon}=A \frac{\sigma^{n}}{d^{p}}
$$

Where $A, n$ and $p$ are temperature-dependent material parameters. Eq. 4 is also employed in the literature [10-12] with different values of $n$ and $p$.

\section{Modelling of the Microstructural and Mechanical Behaviour}

Two steps were carried out to model the material response during high temperature tensile testing. Firstly, the microstructural evolution reported by Mandal et al. [7] was numerically analyzed by calculating the parameters of Eq. 2 and Eq. 3. The fitting of the experimental data for the primary $\alpha$ phase grain size is based on a least-squares approach. An error function was defined in a Matlab script as the sum of the square of the differences between the experimental data and the grain size values from Eq. 1. The parameters $\rho, \mu_{1}, \varphi$ and $\mu_{2}$ in Eq. 2 and Eq. 3 control the value of the error function. The error is minimized by using the 'fminsearch' function of Matlab that has been designed to find the minimum of the unconstrained multivariable functions using the derivative-free method [13]. 
Fig. 1 shows the fitting to experimental grain size data of primary $\alpha$ phase over time for Ti54M alloy at $815{ }^{\circ} \mathrm{C}$ and Ti64 alloy at $925{ }^{\circ} \mathrm{C}$ for a strain rate of $10^{-4} \mathrm{~s}^{-1}$. Experimental grain size data for both materials at no deformation condition and strain rate of $2 \times 10^{-4} \mathrm{~s}^{-1}$ have been employed to fully capture the effect of time (static) and strain rate (dynamic) in the grain growth phenomena.

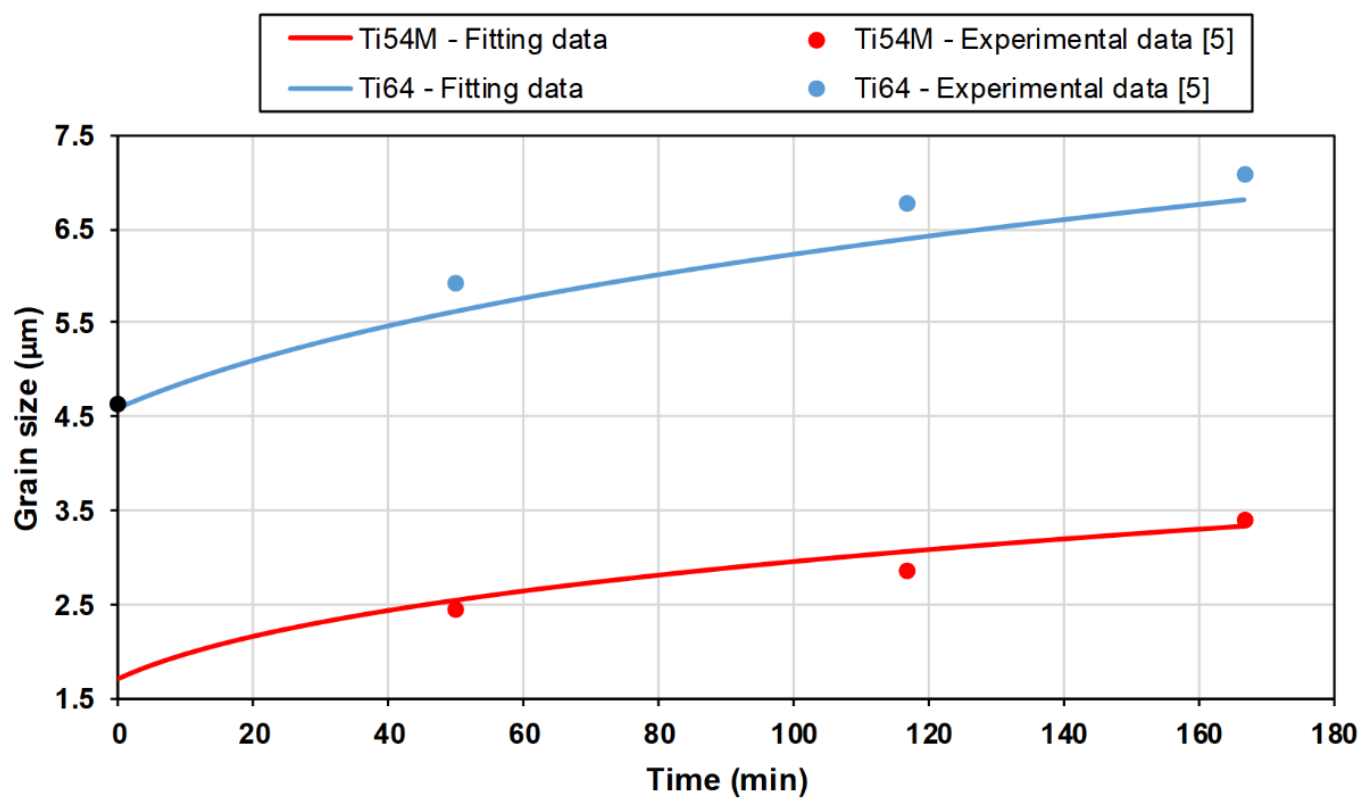

FIG. 1 Grain size evolution of primary $\alpha$ phase over time

Secondly, the flow curves reported by Gomez-Gallegos et al. [8] were fitted by using the grain size evolution data as an input to the constitutive Eq. 4. The fitting of the experimental flow curves was performed based on a similar approach to that previously employed for fitting the grain size data. The model has the parameters $A$ and $p$ to be determined in the curve fitting process while the $n$ value is calculated directly from the $m$ values reported by Gomez-Gallegos et al. [8]. Fig. 2 shows the fitting to the experimental flow curves for Ti54M alloy at $815^{\circ} \mathrm{C}$ and Ti64 alloy at $925^{\circ} \mathrm{C}$ for a strain rate of $10^{-}$ ${ }^{4} \mathrm{~s}^{-1}$. 


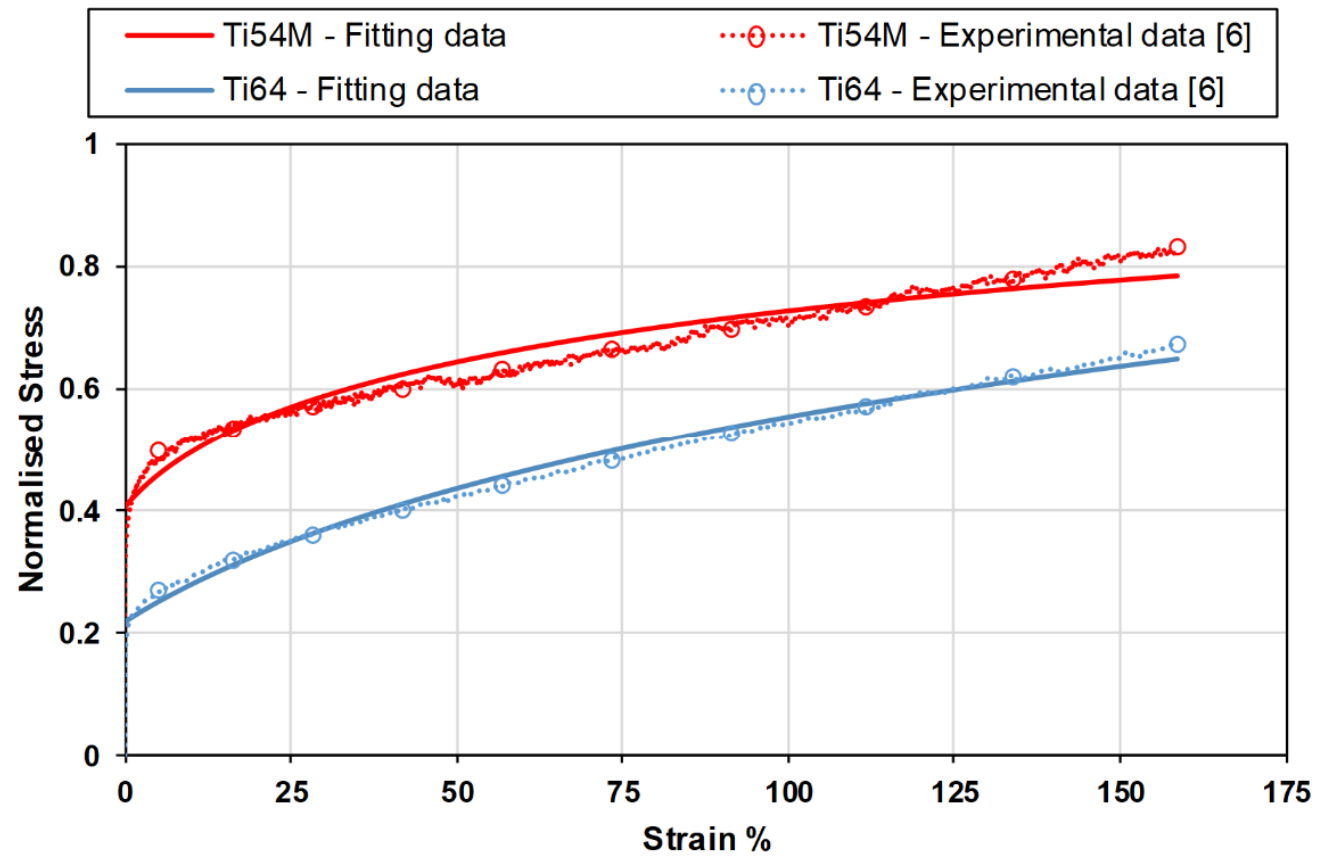

FIG. 2 Flow curves of Ti54M and Ti64 alloys

Fig. 1 and Fig. 2 show good agreement between the experimental and simulation results. Table 1 shows the parameters of the material model for Ti54M alloy at $815^{\circ} \mathrm{C}$ and Ti64 alloy at $925{ }^{\circ} \mathrm{C}$. The values in Table 1 are reported with MPa (stress), mm (length) and s (time) as the units of measure. Fig. 1 also shows that the grain size values of Ti64 alloy are higher than those of Ti54M alloy as the rate of grain growth of Ti64 alloy was higher than that of Ti54M alloy and the initial grain size of Ti64 alloy was greater than that of Ti54M alloy. The initial grain size of Ti64 and Ti54M alloys was $4.6 \mu \mathrm{m}$ and $1.7 \mu \mathrm{m}$, respectively. Fig. 2 also shows that the stresses in Ti54M alloy are higher than those in Ti64 alloy. This is likely due to the lower testing temperature used for the Ti54M alloy.

TABLE 1. Material parameters for Ti54M and Ti64 alloys

\begin{tabular}{ccccc}
\hline Material & $\boldsymbol{\mu}_{\boldsymbol{1}}$ & $\boldsymbol{\mu}_{\boldsymbol{2}}$ & $\boldsymbol{n}$ & $\boldsymbol{p}$ \\
\hline Ti54M & 2.57 & 3.59 & 1.74 & 1.78 \\
Ti64 & 2.76 & 4.45 & 1.92 & 5.47 \\
\hline
\end{tabular}

\section{Modelling the SPF Process for a Non-commercial Part}

The results presented in this paper were used to investigate the behaviour of Ti54M alloy and compare it with the behaviour of Ti64 alloy in an SPF process of a known component geometry. Finite element calculations of the SPF process for Ti54M at $815^{\circ} \mathrm{C}$ and Ti64 at $925^{\circ} \mathrm{C}$ were conducted by implementing the constitutive equations presented in Eq. 1 and Eq. 4 and material parameters in Table 1 into the userdefined subroutine CREEP of Abaqus. Fig. 3 shows the calculated pressure profiles at a controlled strain rate of $5 \times 10^{-4} \mathrm{~s}^{-1}$ for superplastic forming of Ti54M at $815^{\circ} \mathrm{C}$ and Ti64 at $925^{\circ} \mathrm{C}$. Fig. 3 also shows the ratio between the maximum strain rate in the material to the target strain rate of $5 \times 10^{-4} \mathrm{~s}^{-1}$ for superplastic forming of Ti54M at $815^{\circ} \mathrm{C}$ and Ti64 at $925^{\circ} \mathrm{C}$. 


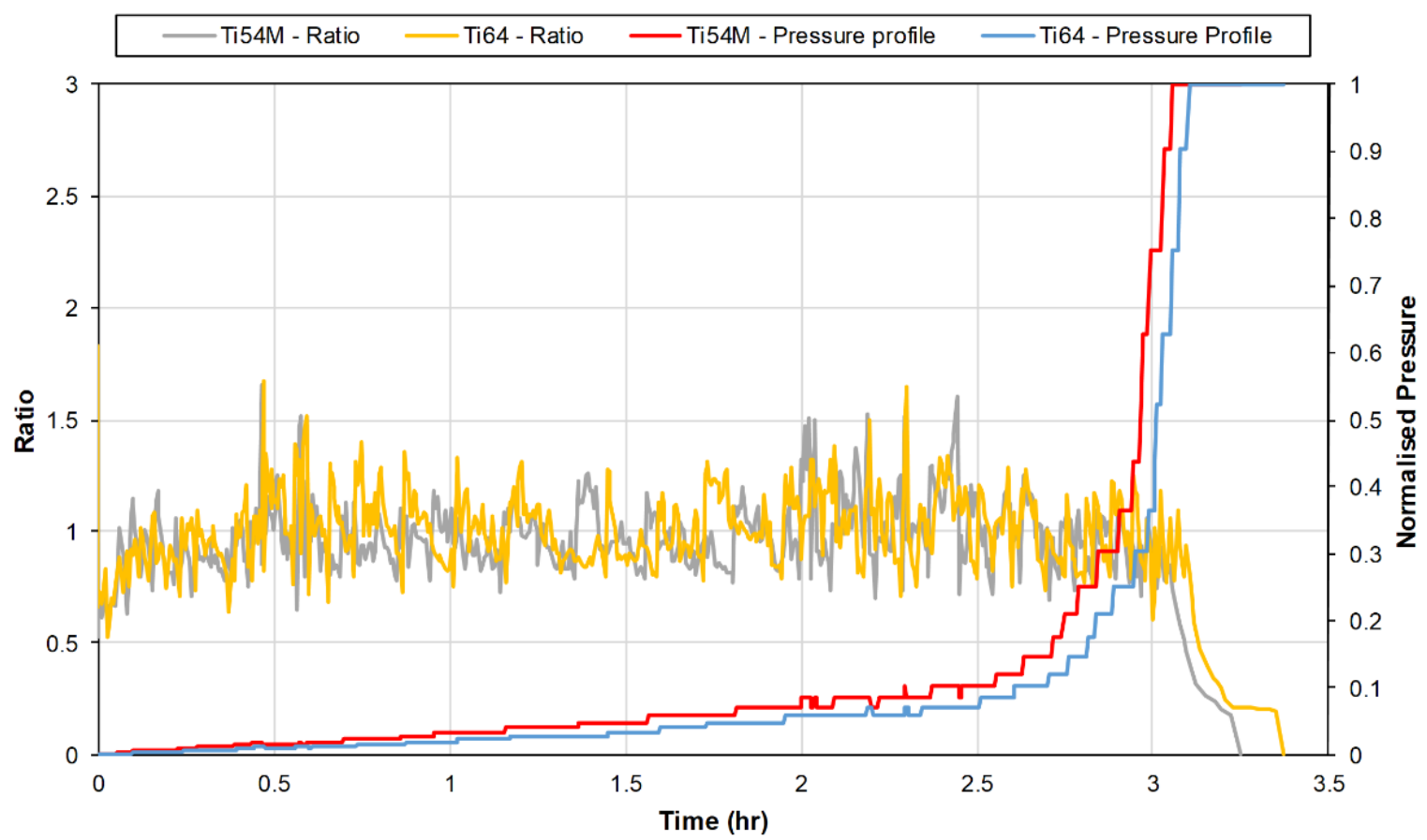

FIG. 3 Pressure profiles and ratio between maximum strain rate to target strain rate for Ti54M and Ti64 alloys under superplastic forming

A total time of $3.25 \mathrm{~h}$ and $3.4 \mathrm{~h}$ was required to completely form the Ti54M and Ti64 parts, respectively. These prolonged times are due to the applied slow controlled strain rate at $5 \times 10^{-4} \mathrm{~s}^{-1}$. It is notable however that the Ti54M part is superplastically formed at a temperature almost $12 \%$ lower than that of the Ti64 part. Forming at lower temperatures will have a great impact on tool life. The current maximum ratio of strain rate to target one was within the range of 0.5 to 1.5 for both alloys during the SPF cycle.

Fig. 4 and Fig. 5 show the grain size distribution of primary $\alpha$ phase for Ti54M alloy at $815{ }^{\circ} \mathrm{C}$ and Ti64 alloy at $925^{\circ} \mathrm{C}$ after $16 \mathrm{~min}$ from the start of the SPF cycle and at the end of the SPF cycle, respectively.

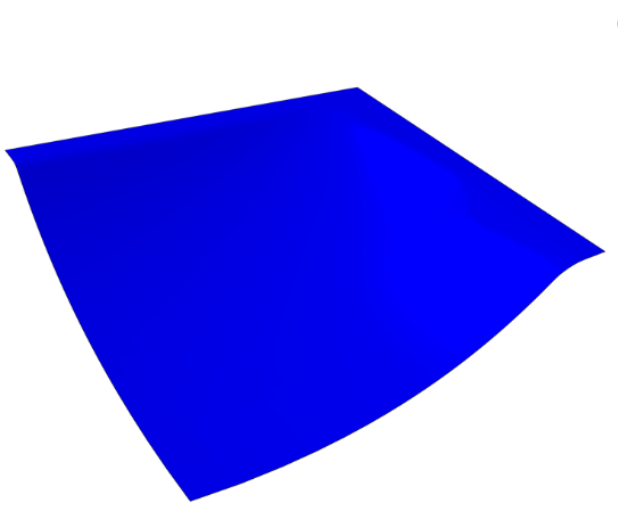

(a)

\section{Grain Size $(\mu \mathrm{m})$}

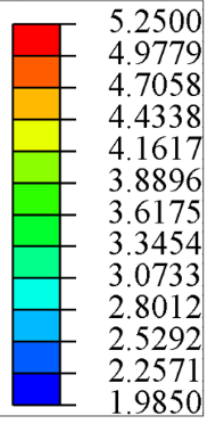

1.9850

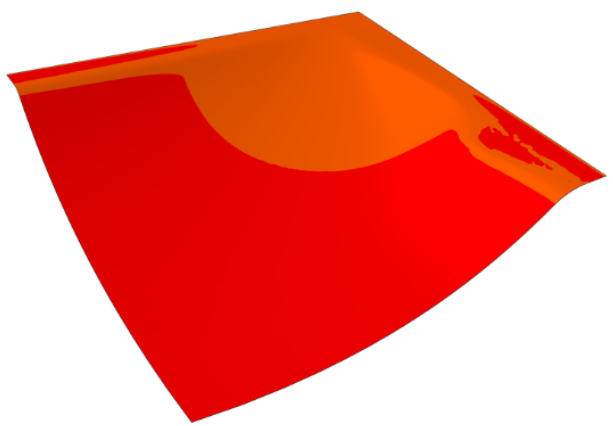

(b)

FIG. 4 Grain size distribution of primary $\alpha$ phase for (a) Ti54M alloy and (b) Ti64 alloy after 16 min from the start of the SPF cycle 


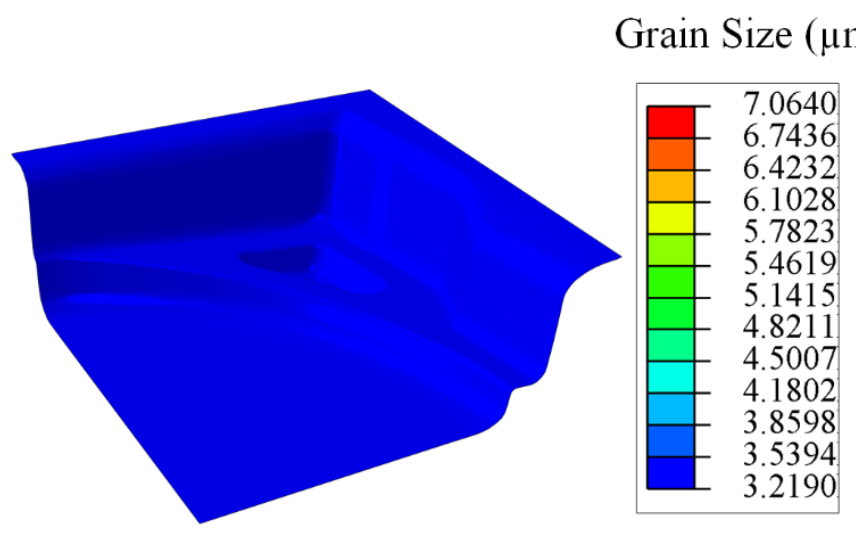

(a)

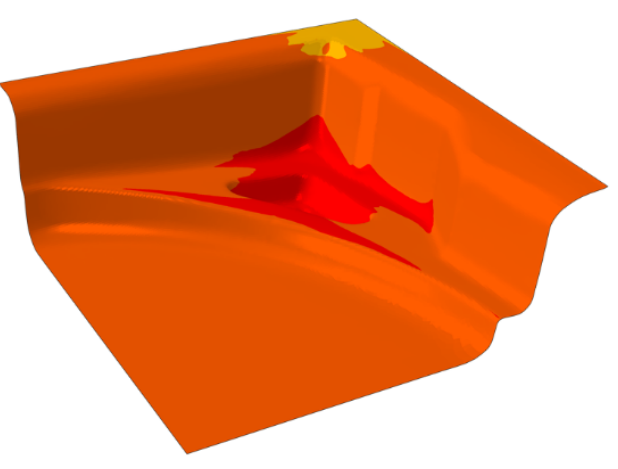

(b)

FIG. 5 Grain size distribution of primary $\alpha$ phase for (a) Ti54M alloy and (b) Ti64 alloy at the end of the SPF cycle

It is notable that the grain size distribution over the Ti64 part is higher than for the Ti54M part as the initial grain size of Ti64 was greater than that of Ti54M as mentioned before.

These results will be used to superplastically form a part and compare against the finite element calculations. Also, more experiments on high temperature tensile tests at different strain rates will be conducted to increase the model's accuracy to capture the effects of strain rate.

\section{Conclusions}

A microstructural-based model was implemented to study the grain size evolution of Ti54M alloy under SPF conditions and its mechanical response. The behaviour of the Ti54M alloy under simulated SPF conditions was compared with that of the Ti64 alloy. The parameters of the models were calibrated via an optimization algorithm defined in Matlab. The fittings agreed well with the experimental data for Ti54M alloy at $815{ }^{\circ} \mathrm{C}$ and Ti64 alloy at $925^{\circ} \mathrm{C}$. Finally, the optimal pressure profile required to superplastically form non-commercial Ti54M and Ti64 parts was obtained following the implementation of the materials models into the commercial FE software Abaqus. The comparison between the behaviour of Ti54M and Ti64 alloys highlighted the capability of Ti54M alloy to be formed via superplastic forming at lower temperatures, which will enable to decrease operational costs due to extended tool life and lower energy consumption.

\section{Acknowledgments}

The authors wish to acknowledge financial support for this work from the Tier 1 members of the Advanced Forming Research Centre, University of Strathclyde (Glasgow, UK) through the Core Research Programme.

\section{References}

[1] T.G. Langdon, Seventy-five years of superplasticity: historic developments and new opportunities, J. Mater. Sci. 44 (2009) 5998-6010. doi:10.1007/s10853-009-3780-5.

[2] V. Velay, H. Matsumoto, V. Vidal, A. Chiba, Behavior modeling and microstructural evolutions of Ti-6Al-4V alloy under hot forming conditions, Int. J. Mech. Sci. 108-109 (2016) 1-13. 
doi:10.1016/J.IJMECSCI.2016.01.024.

[3] M.W. dos Santos, V. Velay, V. Vidal, G. Bernhart, G.F. Batalha, H. Matsumoto, Mechanical behavior of a Ti-6Al-4 V titanium alloy with microstructural evolution modeling under hot and superplastic conditions, Materwiss. Werksttech. 48 (2017) 952-961. doi:10.1002/mawe.201700040.

[4] M. Armendia, A. Garay, L.-M. Iriarte, P.-J. Arrazola, Comparison of the machinabilities of Ti6Al4V and TIMETAL ${ }^{\circledR}$ 54M using uncoated WC-Co tools, J. Mater. Process. Technol. 210 (2010) 197-203. doi:10.1016/J.JMATPROTEC.2009.08.026.

[5] N. Khanna, K.S. Sangwan, Comparative machinability study on Ti54M titanium alloy in different heat treatment conditions, Proc. Inst. Mech. Eng. Part B J. Eng. Manuf. 227 (2013) 96101. doi:10.1177/0954405412466234.

[6] Y. Kosaka, P. Gudipati, Superplasticity and Characterization of TIMETAL ${ }^{\circledR}$ 54M Sheets, Mater. Sci. Forum. 838-839 (2016) 190-195. doi:10.4028/www.scientific.net/MSF.838839.190.

[7] P. Mandal, A. Gomez-Gallegos, D. Gonzalez, P. Blackwell, Superplastic Behaviour of Ti54M and Ti64, in: 14th World Conf. Titan., Nantes, France, 2019.

[8] A. Gomez-Gallegos, P. Mandal, D. Gonzalez, N. Zuelli, P. Blackwell, Studies on Titanium Alloys for Aerospace Application, Defect Diffus. Forum. 385 (2018) 419-423. doi:10.4028/www.scientific.net/DDF.385.419.

[9] J. Lin, T. Zhu, L. Zhan, Constitutive equations for modelling superplastic forming of metals, in: Superplast. Form. Adv. Met. Mater., Elsevier, 2011: pp. 154-183. doi:10.1533/9780857092779.2.154.

[10] E. Alabort, D. Putman, R.C. Reed, Superplasticity in Ti-6Al-4V: Characterisation, modelling and applications, Acta Mater. 95 (2015) 428-442. doi:10.1016/J.ACTAMAT.2015.04.056.

[11] T.G. Langdon, Grain boundary sliding revisited: Developments in sliding over four decades, J. Mater. Sci. 41 (2006) 597-609. doi:10.1007/s10853-006-6476-0.

[12] E. Alabort, P. Kontis, D. Barba, K. Dragnevski, R.C. Reed, On the mechanisms of superplasticity in Ti-6Al-4V, Acta Mater. 105 (2016) 449-463. doi:10.1016/J.ACTAMAT.2015.12.003.

[13] The MathWorks Inc., Matlab User's Guide, Natick, MA, 2015. 